\title{
Face Detection and Tracking in Fuzzy Enhanced Low Contrast Images
}

\author{
G. Sudhavani \\ Professor, \\ Department of ECE, \\ RVR \& JC College of \\ Engineering, Guntur
}

\author{
K.Jaya Ram \\ Student (M.Tech) \\ Department of ECE \\ RVR \& JC College of \\ Engineering, Guntur
}

\author{
P. Venkateswara \\ Rao \\ Associate Professor \\ Department of CSE \\ RVR \& JC College of \\ Engineering, Guntur
}

\author{
K. Satya Prasad \\ Professor, \\ Department of ECE \\ JNTU Kakinada,
}

Kakinada

\begin{abstract}
Face detection and tracking in images have become an important concept for the researchers in the field of Digital Image Processing. Image enhancement refers to the improvement of inner details of image for visual perception. This paper combines these two concepts, using fuzzy logic based enhancement techniques to detect face in low contrast images. After fuzzy enhancement of the input image next step is detection and segmentation of the skin region. Enhancement is based on Fuzzy Intensification Operator (FIO) and Brightness Preserving Dynamic Fuzzy Histogram Equalization (BPDFHE).The detected faces are tracked by enclosing them using two dimensional regions. Comparative study of the enhancement algorithms on face tracking is carried out on the parameters like quality factor, segmentation error, and overlap area.
\end{abstract}

\section{Keywords}

Image enhancement, fuzzy logic, face detection, tracking, bounding box, quality factor, segmentation error, overlap area.

\section{INTRODUCTION}

In digital image processing face detection and tracking [1] [2] have a wide range of applications in various areas like crowed surveillance, artificial intelligence, neural networks, human computer interaction, image monitoring systems etc. There are different algorithms that have been used for face detection and tracking, this paper presents a different approach by combining contrast enhancement [3] [4] with face detection. Enhancement is to improve the perception of information in images for human viewers, and also include concepts of noise removal [5], de-blurring [6], modification of dynamic range of gray-levels etc. Image enhancement is also very helpful in edge detection of images, and those with good contrast may produce better results for edge detection. Enhancement includes two broad categories of techniques spatial and frequency domain methods. The other domain used for enhancement is Fuzzy domain.

Concept of fuzzy logic [7] was introduced in 1965 with the proposal of Fuzzy Set Theory [8] by Lotfi A. Zadeh. Fuzzy logic is a many-valued logic or probabilistic logic that deals with approximate values rather than fixed and exact. Its concepts have been extended to handle the partial truth, where the truth value may vary between true and false. It is a powerful tool to represent and process human knowledge in the form of if-then fuzzy rules. Fuzzy set theory is useful in handling various uncertainties in computer vision and image processing applications. The collection of different fuzzy approaches to image processing that can understand, represent, and process the image is called Fuzzy image processing. Processing an image in fuzzy domain can manage ambiguity and vagueness efficiently. Its applications extend to edge detection and linking [9], image noise removals that are also critical parts of image processing in many computers vision system applications. Edge detection may also provide a tool for segmentation [10] of the image. This segmentation can be achieved in different ways with usage of different color models. A color model [11] is a representation of colors in spatial co-ordinates. Different color models like RGB, HSV, YCC [12] etc. can be used for segmenting the image into meaningful regions that may be processed further depending on the application.

This paper presents a technique to detect and track faces in low contrast images after fuzzy enhancement [13]. Whereas classical face detection and tracking methods generally do that for still images that have good brightness and contrast values. Here fuzzy logic based BPDFHE [14] [15] method and fuzzy intensification operator [2] [3] methods are used for enhancing images. In general a face image has much diversity present and even the image may contain an uncountable nonfacial data. So to separate regions of face from the input image segmentation is a powerful tool and here with the usage of YCC based skin color segmentation is done. A new attempt has been made to use the intuitionist fuzzy set theory in the image enhancement improving the fine details of the image and then track faces using bounding boxes and this does not use complex procedures like neural nets or template matching [16] etc.

\section{PROPOSED ALGORITHM}

The block diagram for proposed algorithm is as shown in figure 1. It consists of input image, fuzzy enhancement, skin color segmentation and detection, binary image conversion, filling the holes, blob detection and then tracking. Input image could be as simple as being given an image that is already in digital form. The system takes a low contrast image as input. After fuzzy enhancement of the image acquired detection and segment the skin color region. Then process proceeds to face detection by converting the image to binary and identify white blobs and eliminate those white pixels that fall under a threshold. The output contains array of rectangles that corresponds to the detected faces, if there are no faces it will be an empty array. 


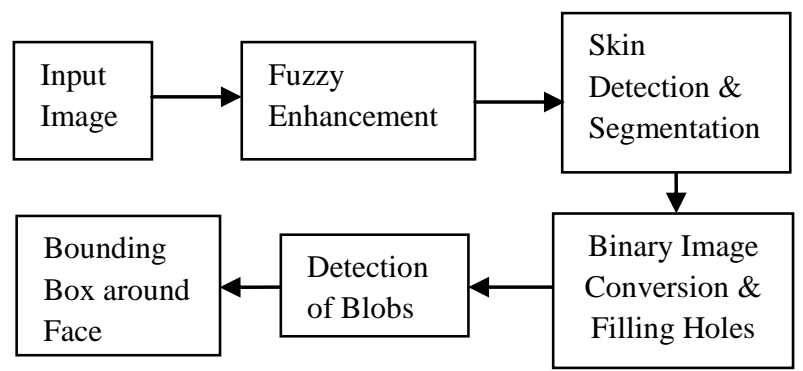

Figure 1: Block Diagram of Proposed Algorithm

\section{IMPLEMENTATION}

\subsection{Fuzzy Enhancement:}

Image enhancement is considered as the transformation of a low clarity image into a high clarity one to make its meaning clearer for human perception and machine analysis. The main goal of contrast enhancement is to process an input image to make the result more clear and suitable than the original image for a specific application.

In image processing techniques care is to be taken, so that the processed image is not conspicuously different from input image. Here fuzzy enhancement techniques used are Brightness Preserving Dynamic Fuzzy Histogram Equalization (BPDFHE) and Fuzzy Intensification Operator (FIO) methods.

\subsubsection{Brightness Preserving Dynamic Fuzzy Histogram Equalization:}

This enhancement technique is formulated in five steps that produce an enhanced image.

Step 1: Image smoothing [17] removes the redundant and noisy maximum and minimum peaks from the image histogram that are generated by high frequency components of the image.

Step 2: Finding the local maximum points by tracing the histogram of the smoothed version of the image.

Step 3: Partition the histogram according to the found maximum points so that the interval is the distance between two successive local maxima.

Step 4: The multiplication length of each interval and the frequency of intensities in that interval is fuzzified by a triangular shaped membership function.

Step 5: This step equalizes the histogram of each interval separately and then normalize the image.

\subsubsection{Contrast Enhancement Using Fuzzy INT- Operator:}

This method uses the intensification operator to decrease the fuzziness of the image that results in the increase of contrast of image. This algorithm is formulated as in the first step separate R, G and B components of the color image and define the parameters of membership function [2] [3]. In the next step fuzzify the gray levels by using the following membership function.

$$
\mu_{\mathrm{mn}}=\mathrm{G}\left(\mathrm{g}_{\mathrm{mn}}\right)\left[1+\frac{\mathrm{g}_{\mathrm{max}}-\mathrm{g}_{\mathrm{mn}}}{\mathrm{F}_{\mathrm{d}}}\right]^{-\mathrm{F}}
$$

Where

$$
\begin{aligned}
& \mathrm{F} \text { - Exponential fuzzifiers } \\
& \mathrm{F}_{\mathrm{d}} \text { - Denomination fuzzifiers } \\
& \mathrm{g}_{\operatorname{mid}} \text { - Mid gray level } \\
& \mathrm{g}_{\max } \text { - Maximum gray level }(\mathrm{L}-1) \text { desired } \\
& \mathrm{g}_{\mathrm{mn}} \text { - Intensity value of }(\mathrm{m}, \mathrm{n}) \text { pixel of input image }
\end{aligned}
$$

$F_{d}$ is calculated by using the below expression.

$$
F_{\mathrm{d}}=\frac{\mathrm{g}_{\max }-\mathrm{g}_{\mathrm{mid}}}{0.5^{\frac{-1}{\mathrm{~F}}}-1}
$$

In the next step membership values are modified and new gray levels are generated. On the application of contrast intensification operator on an image generates a new image with a decreased fuzziness and increased $\mu_{\mathrm{mn}}$ values. Operate every step on the three components separately and concatenate to form the enhanced image.

\subsection{Skin Detection and Segmentation:}

Color is the important feature of human faces and using it body parts can be segmented. Images in which people are fully covered give the best results as the complexity of the algorithm reduces and complex procedures such as neural networks or template matching are not required.

Color is selective emission/reflectance of different wavelengths. All colors seen by humans are identified with combination of three images from the cone cells in our eyes that are sensitive to blue, green and red color. Then all colors can be represented as a vector with three components known as color models that are the spatial representation of colors. The model here used is YCC model. The equations of conversion from RGB to YCC and YCC to RGB are given as:

$$
\begin{aligned}
& \left(\begin{array}{l}
\mathrm{Y} \\
\mathrm{Cb} \\
\mathrm{Cr}
\end{array}\right)=\left(\begin{array}{ccc}
0.257 & 0.504 & 0.098 \\
-0.148 & -0.291 & 0.439 \\
0.439 & -0.368 & -0.071
\end{array}\right)\left(\begin{array}{l}
\mathrm{R} \\
\mathrm{G} \\
\mathrm{B}
\end{array}\right)+\left(\begin{array}{l}
16 \\
128 \\
128
\end{array}\right) \\
& \left(\begin{array}{l}
\mathrm{R} \\
\mathrm{G} \\
\mathrm{B}
\end{array}\right)=\left(\begin{array}{ccc}
1.164 & 0.0 & 1.596 \\
1.164 & -0.392 & -0.813 \\
1.164 & 2.017 & 0.0
\end{array}\right)\left(\begin{array}{l}
\mathrm{Y}-16 \\
\mathrm{Cb}-128 \\
\mathrm{Cr}-128
\end{array}\right)
\end{aligned}
$$

$\mathrm{YCbCr}$ or YCC color model belongs to the family of television transmission systems that comes under BT.601 recommendation. Y component represents to the luminance, $\mathrm{Cb}$ and $\mathrm{Cr}$ represents the chrominance or color components. These $\mathrm{Y}, \mathrm{Cb}$ and $\mathrm{Cr}$ components are shifted and scaled versions of $\mathrm{Y}, \mathrm{U}$ and $\mathrm{V}$ components and these operations are introduced to get the value in the range of $(0,255)$. Threshold values for segmentation of skin color here for $\mathrm{Cb}$ which lies below 70 or above 130 and for $\mathrm{Cr}$ which lies below 133 or above 185 .

\subsection{Face Detection and Tracking}

Face detection step is the backbone of the algorithm and after detecting face regions accurately then they are tracked by using bounding box. This step decides that, which region is most probable human face region by using human face features methods and region properties of human face. There are different methods to detect and track faces like area, Euler number, Eccentricity [1], Centroid [2] etc and sometimes combinations of those methods are also used.

Human faces may contain holes of eyes, nose, mustache etc. due to which sometimes connectivity of regions is missed due to segmentation they seem to be as separate so it becomes difficult to detect faces perfectly. Those regions in the binary image are called white blobs and the idea is to let us identify those white blobs in the image but because of some broken blobs. So the goal now is to fill in such holes and gaps to make those look solid blobs. 
Then eliminate those white pixels that fall under a threshold. If the white pixel density is anything below the threshold is not a face. And such pixels would be discarded and a new binary image would be present with the remaining face pixels. Then it is time to determine the connected components, remove any of the smaller objects present and compute the area of each component. After all the work with elimination of pixels below a pixel density threshold is over it is now easy to put bounding boxes around the detected objects which are nothing but faces. This bounding box is put based on the height to width ratio of the face regions detected above which is a measurable factor using region properties. Parameters of comparison are quality factor, segmentation error and overlap area [10].

\section{SIMULATION RESULTS}

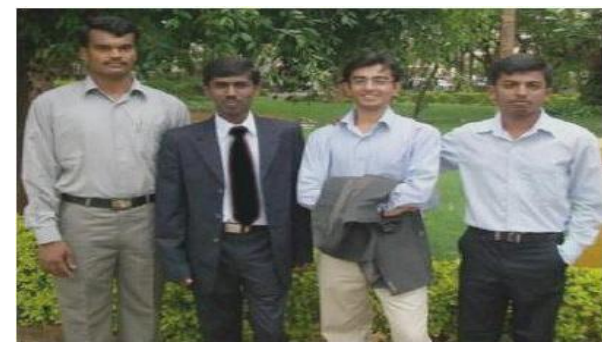

Figure 2: Original Image

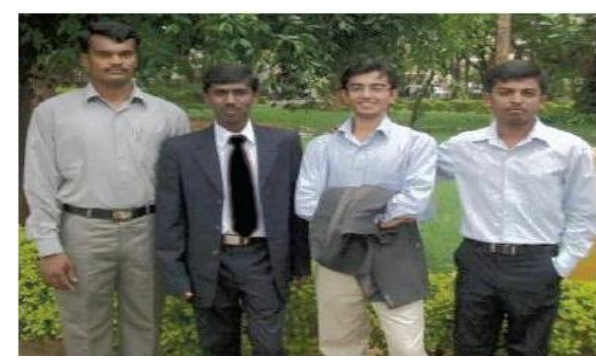

Figure 3: BPD Fuzzy Enhanced Image

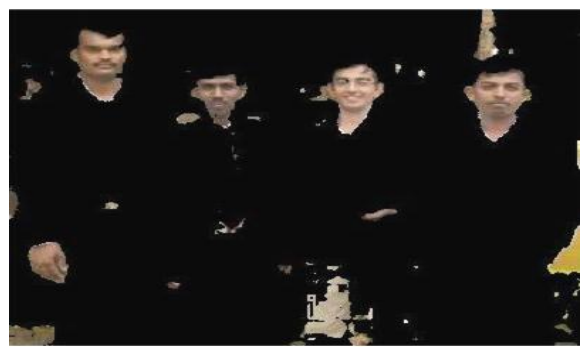

Figure 4: Segmented Skin Color Regions of BPD Fuzzy Enhanced Image

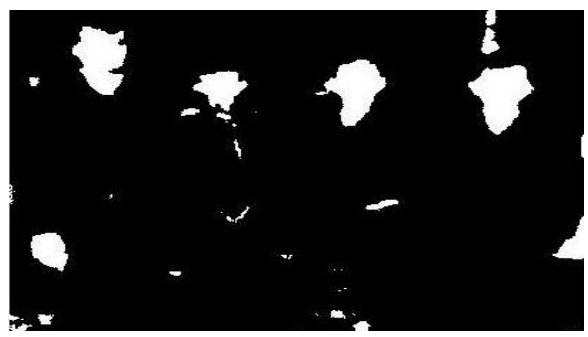

Figure 5: Binary Image after Filling Holes

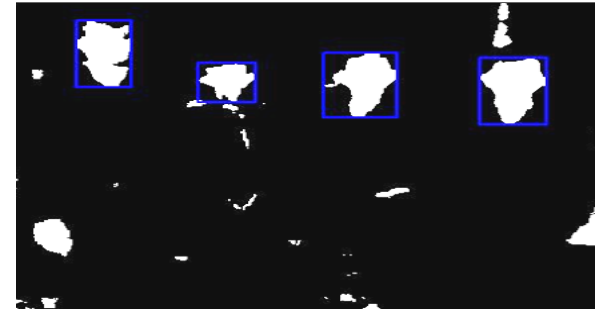

Figure 6: Detected Faces with Bounding Boxes

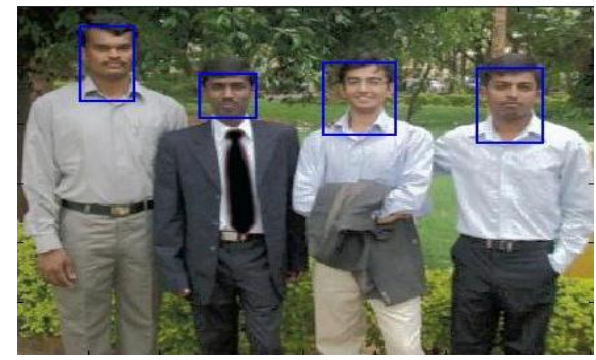

Figure 7: Detected Faces Superimposed on BPD Fuzzy Enhanced Image

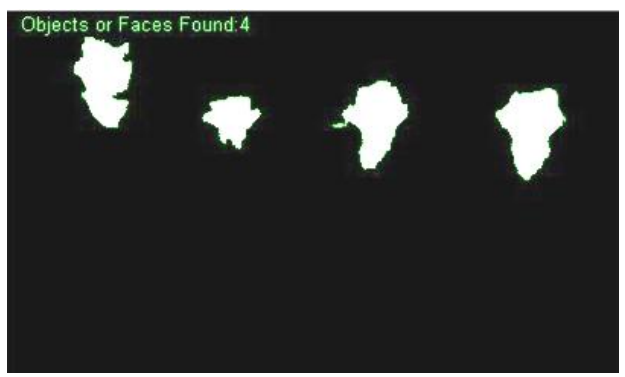

Figure 8: No. of Faces found in BPD Fuzzy Enhanced Image

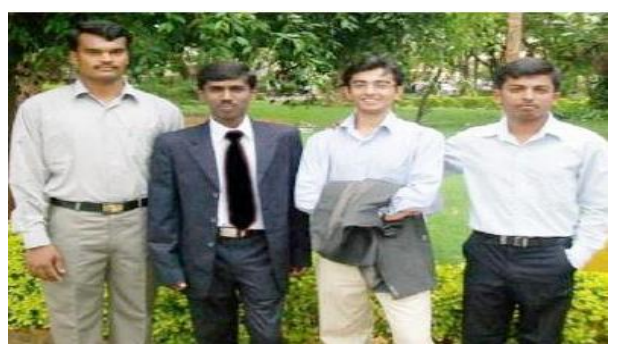

Figure 9: FIO Enhanced Image

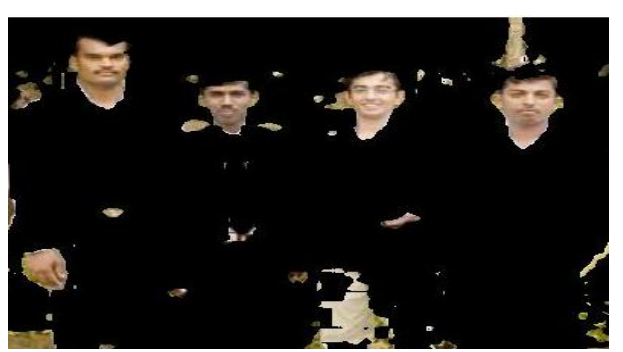

Figure 10: Segmented Skin Color Regions of FIO Image 


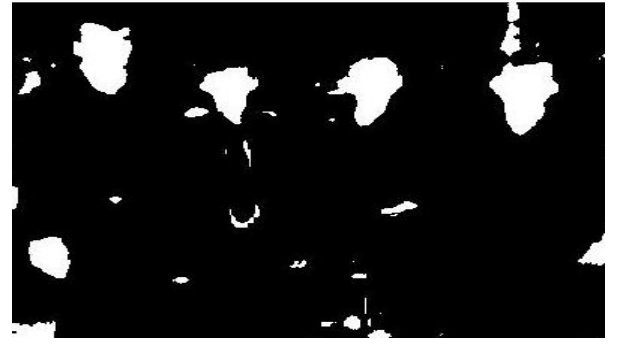

Figure 11: Binary Image after Filling Holes of FIO Enhanced Image

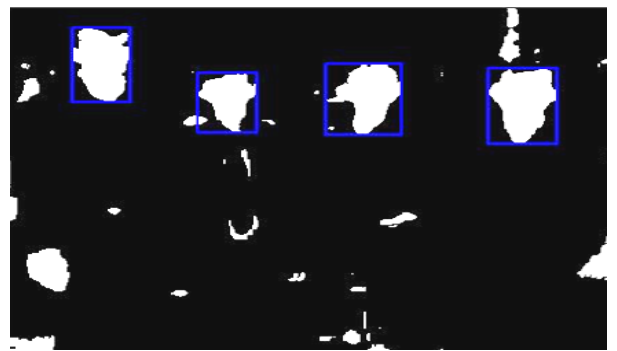

Figure 12: Detected Faces with Bounding Boxes

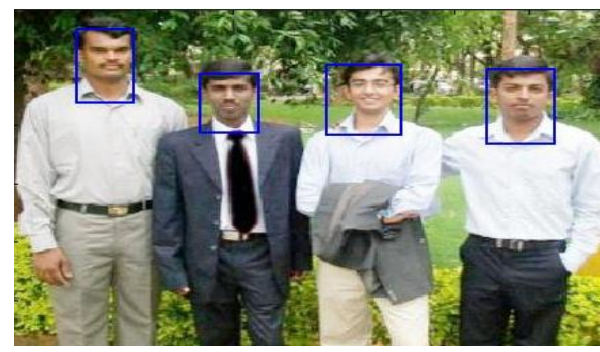

Figure 13: Detected Faces Superimposed on FIO Enhanced Image

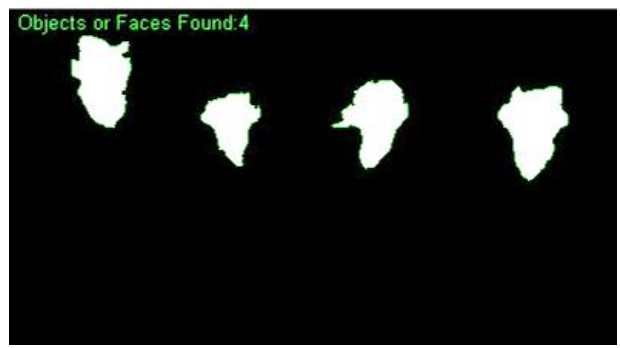

Figure 14: No. of Faces found in FIO Enhanced Image

Table 1: Comparison of time elapsed

\begin{tabular}{|c|c|}
\hline \multicolumn{2}{|c|}{ Comparison of Time Elapsed for Face Detection } \\
\hline $\begin{array}{c}\text { Fuzzy Intensification } \\
\text { Operator }\end{array}$ & $3.746304 \mathrm{Sec}$ \\
\hline $\begin{array}{c}\text { Brightness Preserving } \\
\text { Dynamic Fuzzy Histogram } \\
\text { Equalization }\end{array}$ & $4.425271 \mathrm{Sec}$ \\
\hline
\end{tabular}

\section{Parameters Compared:}

The parameters compared here are Quality factor (QF), Overlap area (OL) and Segmentation error (SE) and their formulae are given below.

$$
\begin{aligned}
& \mathrm{QF}=\frac{2\left(\mathrm{~A}_{1} \bigcap \mathrm{A}_{2}\right)}{\left(\mathrm{A}_{1}+\mathrm{A}_{2}\right)} \times 100 \\
& \mathrm{OL}=\frac{\left(\mathrm{A}_{1} \bigcap \mathrm{A}_{2}\right)}{\left(\mathrm{A}_{1} \bigcup \mathrm{A}_{2}\right)} \\
& \mathrm{SE}=\left(\frac{(\mathrm{OLE}+\mathrm{ILE})}{(2 \times \mathrm{TL})} \times 100\right) \%
\end{aligned}
$$

Where $A_{1}$ is ground truth image, $A_{2}$ is other segmented image which is compared. OLE [9] is the no. of non - face pixels being classified as face pixels, ILE [9] is no. of no face pixels being classified as non - face pixels, and TL is no. of Pixels in the ground truth.

Table 2: Comparison of different parameters

\begin{tabular}{|c|c|c|c|c|c|}
\hline $\begin{array}{c}\text { Method } \\
\text { of Face } \\
\begin{array}{c}\text { Detectio } \\
\mathrm{n}\end{array}\end{array}$ & $\begin{array}{c}\text { Paramete } \\
\mathrm{r}\end{array}$ & $\begin{array}{c}\text { Face- } \\
1\end{array}$ & $\begin{array}{c}\text { Face } \\
-2\end{array}$ & $\begin{array}{c}\text { Face } \\
-3\end{array}$ & $\begin{array}{c}\text { Face } \\
-4\end{array}$ \\
\hline \multirow{3}{*}{ FIO } & QF & 0.062 & $\begin{array}{c}0.07 \\
1\end{array}$ & $\begin{array}{c}0.06 \\
5\end{array}$ & $\begin{array}{c}0.07 \\
0\end{array}$ \\
\cline { 2 - 6 } & $\mathrm{SE}$ & 4.585 & $\begin{array}{c}7.94 \\
0\end{array}$ & $\begin{array}{c}6.62 \\
2\end{array}$ & $\begin{array}{c}6.81 \\
6\end{array}$ \\
\cline { 2 - 6 } & $\mathrm{OL}$ & 6.510 & $\begin{array}{c}7.70 \\
2\end{array}$ & $\begin{array}{c}7.03 \\
8\end{array}$ & $\begin{array}{c}7.55 \\
9\end{array}$ \\
\hline & $\mathrm{QF}$ & 0.054 & $\begin{array}{c}0.05 \\
9\end{array}$ & $\begin{array}{c}0.06 \\
4\end{array}$ & $\begin{array}{c}0.07 \\
2\end{array}$ \\
\cline { 2 - 6 } $\mathrm{BPDFH}$ \\
$\mathrm{E}$ & $\mathrm{SE}$ & 5.612 & $\begin{array}{c}11.1 \\
5\end{array}$ & $\begin{array}{c}6.36 \\
0\end{array}$ & $\begin{array}{c}6.43 \\
2\end{array}$ \\
\cline { 2 - 6 } & $\mathrm{OL}$ & $\begin{array}{c}5.748 \\
6\end{array}$ & $\begin{array}{c}6.03 \\
1\end{array}$ & $\begin{array}{c}6.83 \\
5\end{array}$ & $\begin{array}{c}7.68 \\
4\end{array}$ \\
\hline
\end{tabular}

\section{CONCLUSION}

This paper proposed a technique that performs face detection and tracking of fuzzy enhanced low contrast images. The system takes still colored low contrast images as input, enhance and then face is detected and tracked.

From the visual observations (Fig 3 and Fig 7) it is clear that FIO enhanced image have good visual perception. Also from the observations (Fig 3 to Fig 14) FIO enhanced face detection gave better results than that in the BPDFHE enhanced image.

The comparative study of the two different enhancement algorithms on face tracking schemes is carried out. On the basis of the values of Quality Factor, Overlap Area and Segmentation Error tabulated (Table 2) fuzzy intensification operator enhanced images have yielded convincing results in face detection and tracking compared to that of brightness preserving dynamic fuzzy histogram equalization.

\section{REFERENCES}

[1] G. Prashanth Kumar and M.Shashidhara "Real Time Detection and Tracking of Human Face Using Skin Color Segmentation and Region Properties", International Journal of Signal Processing Systems Vol. 2, No. 2, December 2014.

[2] M. S. Devi and P. R. Baja, "Active facial tracking," in Proc. Third International Conference on Emerging Trends in Engineering and Technology, 2012.

[3] G. Sudhavani, M. Srilakshmi, P. Venkateswara Rao, K. Satya Prasad "Comparison of Fuzzy Contrast Enhancement Techniques" International Journal of 
Computer Applications (0975-8887), vol. 95, No. 22, June 2014

[4] Sudhavani, G., Srilakshmi M., Sravani S., Rao. P.V. "K enhancement of low contrast images using fuzzy techniques", International Conference on Signal Processing and Communication Engineering Systems, 23 Jan. 2015.

[5] G. Sudhavani, G. Madhuri, P. Venkateswara Tao \& Dr. K. Satya Prasad "Additive Noise Removal For Color Images Using Fuzzy Filters", International Journal of Computer Science, Engineering Applications (IJCSEA) vol. 3, No. 3, June 2013.

[6] G. Sudhavani, G. Madhuri, P. Venkateswara Rao \& K. Satya Prasad "Removing of Gaussian Noise from Color Images by Varying Size of Fuzzy Filter", International Journal of Computer applications (09751 - 8887), vol. 72, No. 17, June 2013.

[7] Etiene E. Kerre and Mike Nachtegael "Fuzzy Techniques in Image Processing" Studies in fuzziness and soft computing, ISSN 1434-9922, 2000.

[8] W. Pedrycz, F. Gomode "An Introduction to Fuzzy Sets: Analysis and Design", the MIT Press, 1998.

[9] G. Sudhavani, S. Sravani, P. Venkateswara Rao \& K. Satya Prasad "Fuzzy Edge Linking Process on Fuzzy Noise Filtered Image", International Journal of Computer Applications (0975-8887), vol. 93, No. 15, May 2014.
[10] G. Sudhavani \& Dr. K.Satya Prasad "Segmentation of Lip image by Modified Fuzzy C-means Clustering Algorithm" International Journal of Computer Science \& Network Security, vol. 9, No. 4, April 2009.

[11] "Video Processing And Communications" by Yao Wang, Joern Ostermann, and Ya-Qin Zhang.

[12] Marius, Diedrick; Pennathur, Sumita; Rose, Klint, "Face Detection Using Color Thresholding and Eigenimage Template Matching".

[13] R. C. Gonzalez, R. E. Woods, Digital Image Processing”, 3rd Ed., Prentice Hall.

[14] Haidi Ibrahim, Nicholas Sia Pik Kong, "Brightness Preserving Dynamic Histogram Equalization for Image Contrast Enhancement", IEEE Trans. Consumer Electron, Vol. 53, no. 4, Nov 2007.

[15] M. Abdullah-Al-Wadud, Md. Hasanul Kabir, M. Ali Akber Dewan, and Oksam Chae, "A Dynamic Histogram Equalization for Image Contrast Enhancement”, IEEE Trans. Consumer Electron, vol. 53, May 2007.

[16] Rajandeep Kaur, Vijay Dhir, "Fuzzy Logic Based Novel Method of Face Detection" International Journal of Latest Research in Science and Technology Volume 2, January-February (2013).

[17] Sankar K. Pal, Robert A. King, "Image Enhancement using Smoothing with Fuzzy Sets", IEEE Transactions on Systems, Man and Cybernetics, vol. SMC-11, No. 1, pp. 494-501, July 1981. 